\title{
Metodología de aprendizaje del sistema numérico binario basado en teoría de aprendizaje por descubrimiento*
}

\author{
|Ómar Iván Trejos Buriticá**
}

Recibido: 27/06/2016 - Aceptado: 13/09/2017

https://doi.org/10.22395/rium.v17n33a7

\begin{abstract}
Resumen
En el presente artículo se examinan los posibles cambios en el aprendizaje autónomo de estudiantes de primer semestre de Ingeniería de Sistemas y Computación a partir de la aplicación de la teoría de aprendizaje por descubrimiento, específicamente en la asimilación, apropiación, aplicación y retroalimentación del sistema numérico binario. La metodología utilizada corresponde al marco de la investigación educativa con carácter cualitativo, articulada con un estudio de casos simple, individual y múltiple. La investigación inicia con el anuncio de la teoría de aprendizaje que se va a utilizar y finaliza con la evaluación y retroalimentación entre docente y estudiantes a partir de los resultados cualito-cuantitativos obtenidos. Se utilizó como herramienta de apoyo la hoja electrónica Excel y se hizo un trabajo de segmentación del grupo de forma que se pudieran establecer criterios comparativos entre la metodología tradicional de enseñanza del sistema binario y la metodología basada en aprendizaje por descubrimiento. Los resultados evidencian un aprendizaje más significativo y con sentido por parte del grupo a quien se le aplicó la teoría del aprendizaje por descubrimiento. De la misma forma, se notó mayor independencia académica por parte de ese subgrupo de estudiantes y una confianza mayor en relación con el abordaje de temas desconocidos pero enmarcados dentro del conocimiento disciplinar propio de la Ingeniería de Sistemas.
\end{abstract}

Palabras clave: aprendizaje; aprendizaje por descubrimiento; metodología de aprendizaje; sistema binario; sistema numérico.

\footnotetext{
Artículo derivado del proyecto de investigación Desarrollo de un modelo metodológico para el aprendizaje de la programación imperativa en Ingeniería de Sistemas basado en aprendizaje significativo, aprendizaje por descubrimiento y en el modelo $4 q$ de preferencias de pensamiento, identificado con el código interno 6-16-13 de la Universidad Tecnológica de Pereira en Colombia y ejecutado entre enero de 2015 y diciembre de 2016.

** Ph. D., Universidad Tecnológica de Pereira, Facultad de Ingenierías. Carrera 27 N. ${ }^{\circ}$ 10-02, teléfono (+57) 63137300. Correo electrónico: omartrejos@utp.edu.co. Orcid: https://orcid.org/0000-0002-3751-6014
} 


\title{
Learning Methodology of Binary Number System Based on Discovery Learning Theory
}

\begin{abstract}
This article examines the possible changes in the autonomous learning of first academic term students of Computer Science Engineering from the application of the theory of learning by discovery, specifically in the assimilation, appropriation, application and feedback of the binary numerical system. The methodology used corresponds to the framework of qualitative educational research, articulated with a simple, individual and multiple case study. The research begins with the announcement of the learning theory to be used and ends with the evaluation and feedback between teacher and students based on the quality-quantitative results obtained. The Excel spreadsheet was used as a support tool and the group was segmented so that comparative criteria could be established between the traditional teaching methodology of the binary system and the methodology based on learning by discovery. The results show a more significant and meaningful learning on the part of the group to which the theory of learning by discovery was applied. In the same way, it was noted greater academic independence on the part of this subgroup of students and a greater confidence in relation to the approach of unknown topics but framed within the disciplinary knowledge proper to Computer Science Engineering.
\end{abstract}

Keywords: learning; learning by discovery; learning methodology; binary system; numerical system.

\section{Metodologia de aprendizagem do sistema númerico binário baseada na teoria de aprendizagem por descoberta}

\begin{abstract}
Resumo
No presente artigo, as possíveis mudanças na aprendizagem autônoma de estudantes do primeiro semestre de Engenharia de Sistemas e Computação são examinadas a partir da aplicação da teoria de aprendizagem por descoberta, especificamente na assimilação, apropriação, aplicação e retroalimentação do sistema numérico binário. A metodologia utilizada corresponde ao âmbito da pesquisa educativa com caráter qualitativo, articulada com um estudo de casos simples, individual e múltiplo. A pesquisa inicia com o anúncio da teoria de aprendizagem que será utilizada e finaliza com a avaliação e retroalimentação entre docente e estudantes a partir dos resultados qualitativos e quantitativos obtidos. A folha eletrônica Excel foi utilizada como ferramenta de apoio e realizou-se um trabalho de segmentação do grupo de forma que fosse possível estabelecer critérios comparativos entre a metodologia tradicional de ensino do sistema binário e a metodologia baseada em aprendizagem por descoberta. Os resultados evidenciam uma aprendizagem mais significativa e com sentido por parte do grupo no qual se aplicou a teoria da aprendizagem por descoberta. Da mesma forma, notou-se maior independência acadêmica por parte desse subgrupo de estudantes e uma maior confiança com respeito à abordagem de temas desconhecidos, mas pertencentes ao conhecimento disciplinar próprio da Engenharia de Sistemas.
\end{abstract}

Palavras-chave: aprendizagem; aprendizagem por descoberta; metodologia de aprendizagem; sistema binário; sistema numérico. 


\section{INTRODUCCIÓN}

Alrededor de la enseñanza y el aprendizaje existen diversas teorías y modelos que pueden plantearse desde las ópticas más tradicionales hasta las más innovadoras [1]. Los docentes universitarios, desde diferentes enfoques, estilos y experiencias, han validado el hecho de que la práctica docente no puede establecer un modelo único que interprete y aplique de manera fidedigna toda su fundamentación teórica dado que mucho del quehacer docente depende de lo que sucede en el aula, el contexto escolar y extraescolar de cada uno de los estudiantes, la interacción y el nivel de aproximación y comunicación que se dé entre docente y estudiante, y otros factores que solo surgen cuando un curso ha comenzado y se va desarrollando a lo largo del tiempo [2].

Ha sido, pues, la enseñanza de la programación de computadores un espacio en donde múltiples enfoques, tendencias y visiones han coexistido en la búsqueda de propender por servir al estudiante el conocimiento tecnológico de manera que pueda ser asimilado por un camino cada vez más expedito. Tanto los enfoques didácticos como la orientación han establecido en la actualidad un marco suficientemente diverso que permite que el docente de programación de computadores pueda enriquecerse y nutrirse de una forma como nunca antes había sido posible en favor del logro de los objetivos de aprendizaje [3].

Por su parte, las matemáticas por su naturaleza problémica, siempre han ofrecido pasivamente un espacio de aplicación de la lógica y, de su mano, la búsqueda de soluciones a los problemas que las mismas matemáticas proveen. La tendencia a resolver problemas matemáticos a través de las posibilidades que brinda la programación de computadores se ha ido convirtiendo poco a poco en una necesidad pues, de esta forma se establecen nexos temáticos entre dos áreas de conocimiento fundamentales, en tiempos modernos, para el estudiante de ingeniería de sistemas y computación: matemáticas y programación, puesto que aquella provee los problemas y la forma de presentarlos formalmente, y esta provee posibles soluciones (para algunos de ellos) que pueden ser implementados a la luz de la tecnología computacional moderna [4]. Es conveniente aclarar que no todos los problemas que proveen las matemáticas son solucionables (o fácilmente solucionables) a través de la programación de computadores.

Tres modelos didácticos han imperado en la enseñanza de la programación [5]: el modelo llamado de la escalera semiótica que a partir del uso de lenguajes de programación e incorporación de instrucciones identifica una secuencia lógica bajo un marco sintáctico, con unos elementos semánticos y dentro de un contexto pragmático del lenguaje como su gran herramienta; el segundo modelo puede describirse a partir de objetivos de taxonomía cognitiva que categoriza las soluciones desde una clasificación 
similar a la que fuera planteada y formulada como objetivos de Bloom; finalmente, el tercer modelo es el de la resolución de problemas que se aproxima mucho más a un modelo que fortalece el proceso de aprendizaje que a la estrategia misma de enseñanza [6].

Sin que sean la panacea, pero sabiendo que son fundamentos que propenden por facilitar el trabajo del docente universitario, es claro que estos modelos brindan una base sobre la cual la enseñanza de la programación puede acudir a una amalgama de ellos para lograr lo mejor en pro del proceso de aprendizaje por parte del estudiante. En estos modelos, de todas formas, subyacen dificultades en el aprendizaje de la programación, dificultades mismas en la enseñanza que cuestionan la necesidad de acudir a ellos o, mejor, de plantear las soluciones desde lo puramente formal tal como lo proveen las matemáticas, la necesidad de refinar y sintonizar los entornos de aprendizaje así como la competencia lectora que permita resolver problemas a partir de su correcta interpretación [7].

Este artículo es, precisamente, un ejemplo de la forma como se puede proponer una estrategia de enseñanza que acuda a lo mejor de estas situaciones tanto en lo didáctico como en lo práctico, lo académico y lo aplicativo, lo formal y lo tecnológico.

De otra parte, debe tenerse en cuenta que si la programación puede proveer soluciones a problemas que la matemática propone, entonces no es suficiente con que se llegue a un nivel excelso de conocimiento en la sintaxis y en los recursos del lenguaje de programación ni tampoco con que se conozca, con profundidad, la notación matemática y la expresión formal de planteamiento de los problemas [8]. Se requiere de ambos componentes para que la solución pueda visualizarse por parte del estudiante, mediados por una lógica de desarrollo de algoritmos y construcción de programas que permitan que ambas áreas de conocimiento puedan entenderse tal como se presenta en la figura 1.

Los resultados de la investigación ponen de presente que, en los procesos de aprendizaje de la programación, y específicamente en la resolución de problemas derivados de las matemáticas, no es suficiente el nivel sintáctico tecnológico, sino que debe acompañarse de un conocimiento conceptual junto con el uso de habilidades orientadas a la resolución de problemas computables, procesos de prueba, depuración de errores lógicos y puesta a punto del programa. Igualmente, se indica la importancia de poder mejorar en los alumnos la necesidad de la representación de modelos mentales, la descomposición de una solución algorítmica en unidades más simples y la autoevaluación de la apropiación de la lógica computacional en cada estudiante [9]. 


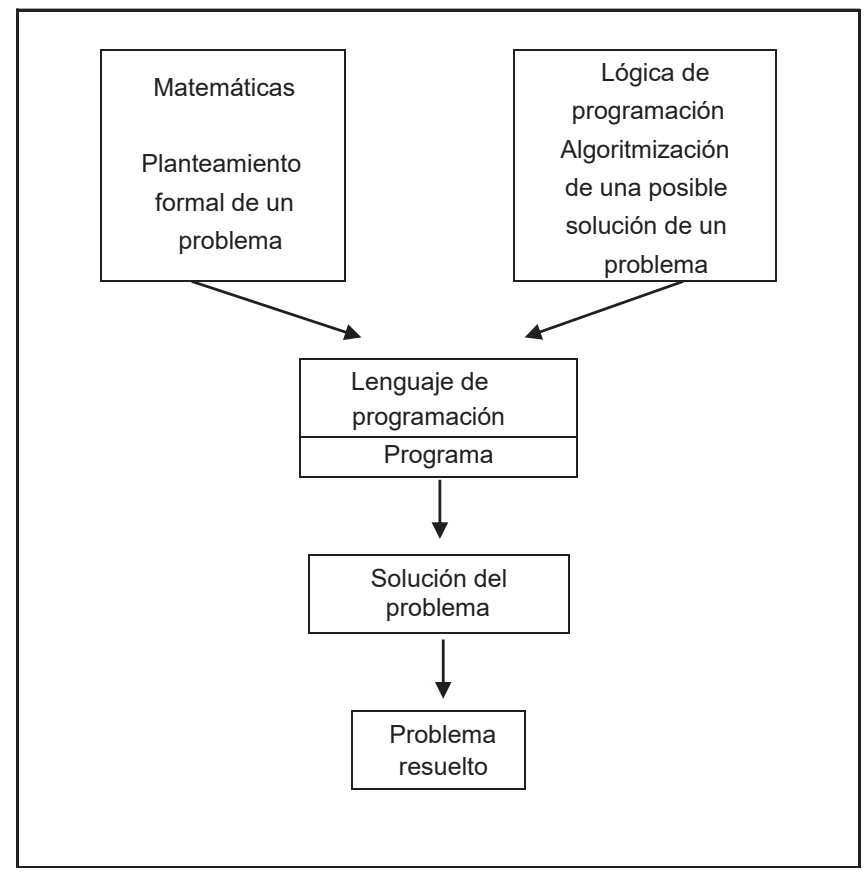

Figura 1. Relación matemáticas-lenguajes de programación Fuente: elaboración propia

La problemática abordada en el presente artículo hace referencia a la necesidad de apoyarse en teorías de aprendizaje por parte de docentes de las áreas de ingeniería para hacer más efectiva su labor [10] y más alcanzables los logros de aprendizaje por parte de sus estudiantes. El aprendizaje del sistema numérico binario apoyado en la teoría del aprendizaje por descubrimiento, tal como lo evidencian los resultados, favorece el aprendizaje autónomo con responsabilidad, incrementa el nivel de comprensión y de análisis, posibilita la colaboración entre estudiantes y permite que ellos mismos construyan significado y sentido a partir de sus propias experiencias y elaboraciones mentales.

¿Es posible desarrollar una metodología que permita entender, apropiar, asimilar, aplicar y retroalimentar el sistema numérico binario a partir de la teoría de aprendizaje por descubrimiento? La respuesta a esta pregunta de investigación está contenida en el presente artículo que confirma no solo lo propuesto sino que, a partir de la opinión de los mismos estudiantes, plantea una estrategia que, llevada apropiadamente, puede llegar a optimizar el tiempo de aprendizaje en los estudiantes en estas temáticas. Fundamentalmente, el marco de referencia de esta investigación lo provee la teoría de aprendizaje por descubrimiento en un contexto tecnológico [11] y [12] y la teoría de 
aprendizaje significativo [13] y su aplicación en el campo de la ingeniería de sistemas en el área de programación de computadores en la resolución de problemas matemáticos. La posición epistemológica de estos enfoques acude tanto a la necesidad consciente de crear en los estudiantes las capacidades de investigar de manera autónoma [14], de descubrir a partir de una buena fundamentación, de inferir y apropiar nuevos conocimientos y de aprender tomando parte activa en su proceso de aprendizaje lo cual incluye autoevaluarse y automejorarse.

Este artículo es uno de los productos asociados al proyecto de investigación Desarrollo de un modelo metodológico para el aprendizaje de la programación imperativa en Ingeniería de Sistemas basado en aprendizaje significativo, aprendizaje por descubrimiento y el modelo $4 Q$ de preferencias de pensamiento aprobado por la Vicerrectoría de Investigaciones, Innovación y Extensión de la Universidad Tecnológica de Pereira bajo el código 6-16-13.

\section{TEORÍA}

En términos matemáticos, un sistema numérico se puede definir como un conjunto de símbolos y reglas que pueden ser usados para representar, tanto datos numéricos como cantidades. Los sistemas numéricos se caracterizan por su base que es el valor que indica el número de símbolos distintos con los cuales se pueden representar las cantidades numéricas. Esa misma base es también el coeficiente que sirve para determinar el valor que corresponde a cada símbolo dependiendo de la ubicación que ocupe dentro de un número.

En las áreas de la aritmética, el álgebra, así como en el análisis matemático, un sistema numérico corresponde a un conjunto definido que está provisto de dos operaciones que, al tiempo, son las que verifican las condiciones relacionadas con tres leyes: la ley conmutativa, la ley asociativa y la ley distributiva. Esto nos da a entender que los sistemas numéricos poseen una estructura algebraica (la cual puede estar definida entre los conceptos de álgebra sobre un cuerpo monoide, anillo o estructuras modernas) [15]. Estos sistemas también satisfacen lo que en matemáticas se conocen como propiedades de orden, es decir, si son de buen orden o son de orden total y también tienen propiedades topológicas adicionales.

Desde una perspectiva más general, como lo posibilita la matemática, un conjunto que posea adición y multiplicación puede considerarse como un sistema numérico y es por ello que se incluyen entre estos a los números naturales, los números enteros, los números racionales, los números reales y los números complejos, sin desconocer que existen conjuntos que generalizan algunos de los sistemas numéricos enunciados anteriormente. Formalmente, tal como lo provee la notación matemática, no se ha 
formulado una definición formal de lo que es un sistema numérico, pero se ha encontrado que todos los sistemas numéricos con operaciones binarias tienen propiedades comunes entre sí. Las dos operaciones que se definen son la adición y la multiplicación sin desconocer que la multiplicación es la expresión distributiva de la adición o que la multiplicación es una operación que consiste en la expresión de un conjunto de sumas abreviadas en su notación formal [16].

El sistema binario (que también se conoce como sistema diádico) se utiliza mucho en las ciencias de la computación y es un sistema de numeración en el cual los números se representan usando solamente dos dígitos: el uno (1) y el cero (0) [17]. A nivel de representación electrónica, es el sistema que se utiliza en los computadores dado que permite una asociación con los niveles de voltaje que, internamente, posibilita la representación de los datos.

Debe advertirse que, a nivel conceptual, en materia de computadoras, no es el único sistema que se utiliza en ciencias de la computación para referenciar determinados valores pues también están el sistema octal y el sistema hexadecimal. Un valor 1 representa la presencia de un voltaje específico y un valor 0 representa la ausencia de dicho voltaje (o para ser más precisos, representa un voltaje significativamente menor). Debido a esto, el valor 1 se asocia con un estado encendido o un voltaje alto y el valor 0 se asocia con un estado apagado o un voltaje bajo [18]. Existe una analogía directa entre el sistema decimal y el sistema binario.

Si bien la definición formal de sistema numérico establece que es suficiente con que se tengan definidas las operaciones de adición y multiplicación, es cierto también que las operaciones que aquí se describen constituyen el corpus complejo de las relaciones básicas que se pueden establecer entre estos sistemas de representación numérica. A nivel electrónico, el sistema binario solamente posibilita la adición. La multiplicación es un conjunto de adiciones sucesivas, la resta se implementa basándose en la suma y la división es un conjunto de restas sucesivas.

La teoría de aprendizaje por descubrimiento fue formulada y desarrollada por el pedagogo y sicólogo norteamericano Jerome Seymour Bruner dentro de un marco de índole constructivista que también se conoce como aprendizaje heurístico. Lo principal que se destaca de esta teoría es que se busca promover en el alumno (también conocido como aprendiente) la capacidad de adquirir los conocimientos por sí mismo [19] a partir de los nuevos conocimientos del saber disciplinar específico.

La teoría propone que, a partir de lo que el docente expone al estudiante, este debe descubrir progresivamente lo que falta o lo que sigue [19] y, de esta forma, ejerciendo su labor de descubrimiento, el conocimiento quedará ubicado en la memoria a largo plazo 
pues tiene un significado y un sentido superior producto del esfuerzo del estudiante por acceder a él. El concepto de significado le confiere una utilidad adicional al nuevo conocimiento y por ello, aprendizaje por descubrimiento y aprendizaje significativo, son dos teorías que se requieren para lograr efectividad en la labor docente en propuestas como la que se expone en este artículo.

A juicio de Bruner, los estudiantes podían aprender mucho más si el proceso de aprendizaje es producto de un descubrimiento guiado que provenga de la exploración promovida por la curiosidad y la fascinación, dos elementos que el autor consideraba de vital importancia para generar motivación (tercer componente del aprendizaje significativo) y de esta forma poder aproximarse al aprendizaje autónomo [13]. Bruner también establece que el docente no tiene como papel, en el aula, la explicación de unos contenidos desde el inicio hasta su último tema, sino que debe buscar y aplicar estrategias que permitan estimular a sus estudiantes a través de mecanismos de observación, comparación, desarrollo de la capacidad de análisis, hallazgo de similitudes y divergencias y otros elementos que son los que, a la postre, permitirán descubrir.

Entre las ventajas que se han endilgado a la aplicación de la teoría de aprendizaje por descubrimiento, está el hecho de permitir la superación de las limitantes que implican las estrategias de aprendizaje tradicional (memorístico o mecanicista), facilitar la estimulación en los estudiantes para pensar por sí solos planteando hipótesis y tratando de verificarlas a través de métodos sistemáticos y aumentar la autoestima y la sensación de seguridad en los alumnos a partir de la búsqueda de soluciones creativas frente a problemas planteados.

Entre los críticos más enconados de la teoría del aprendizaje significativo se encuentra el también norteamericano David Paul Ausubel, quien aseguraba que existía una falsa visión sobre los beneficios de esta teoría dado que, según este autor, no necesariamente el aprendizaje por descubrimiento es absolutamente significativo ni la estrategia de aprender por recepción (memorístico) es necesariamente mecánica [13]. Todo dependerá de la información que captan los sentidos y procesa el cerebro sean almacenados dentro de la estructura individual cognitiva de cada estudiante.

\section{METODOLOGÍA}

Se ha acudido a estas teorías planteadas debido a que se encuentra gran relación entre sus planteamientos teóricos con el corpus académico que sustenta la teoría de la programación. La teoría del aprendizaje significativo ha sido útil para relacionar los conceptos de sistema numérico y, específicamente, de sistema binario con situaciones prácticas de la vida cotidiana que forman parte del contexto extrainstitucional de los estudiantes. 
Por su parte, la teoría de aprendizaje por descubrimiento ha permitido que, tomando como base la fundamentación propia tanto del sistema decimal como del sistema "ternario", el estudiante pueda inferir los conceptos propios del sistema binario tal que pareciera haberlo descubierto en los términos en los que las matemáticas los plantean. El sistema binario por lo tanto se convierte en un constructo propio del estudiante lo cual lo ubica, como objeto de conocimiento, en un espacio de memoria a largo plazo que le permite acudir a él con mayor facilidad que si se expone a los estudiantes en los términos tradicionales de la clase magistral [20].

La presente investigación se realizó con los grupos de Programación I del programa Ingeniería de Sistemas de la Universidad Tecnológica de Pereira durante los semestres académicos de los años 2014, 2015 y 2016 tal como se muestran en la tabla 1, con un total de 92 estudiantes.

Tabla 1. Estudiantes que participaron en la Investigación

\begin{tabular}{|c|c|c|}
\hline \multirow{2}{*}{ Año } & Semestre & $\begin{array}{c}\text { Cantidad de } \\
\text { estudiantes }\end{array}$ \\
\hline \multirow{2}{*}{2014} & I & 19 \\
\cline { 2 - 3 } & II & 22 \\
\hline \multirow{2}{*}{2015} & I & 21 \\
\cline { 2 - 3 } & II & 20 \\
\hline \multirow{2}{*}{2016} & I & 21 \\
\cline { 2 - 3 } & II & 19 \\
\hline \multirow{2}{*}{} & \multicolumn{2}{|c|}{ Total } \\
\hline \multirow{2}{*}{ Fuente: elaboración propia } \\
\hline
\end{tabular}

La metodología que se desarrolló para la presente investigación consistió en siete fases que se describen en la tabla 2. Es de anotar que, para los efectos de obtener unos resultados más objetivos, se socializó con los estudiantes la metodología que se iba a adoptar, previo al inicio del proceso.

En relación con la forma como se realizó la aplicación de la teoría de aprendizaje por descubrimiento en el tema del sistema binario, la tabla 3 muestra la estrategia adoptada. Cada grupo se dividió en dos subgrupos de manera que con uno se realizó la exposición magistral tradicional indicando las características del sistema binario tal como se acostumbra a hacer en estos cursos. Con el otro subgrupo se realizó una exposición magistral sobre la base de un sistema llamado quinquenario, cuya base es 5 . 
Tabla 2. Descripción de fases de la metodología

\begin{tabular}{|c|c|c|}
\hline Fase & Nombre & Descripción \\
\hline 1 & Preámbulo & $\begin{array}{l}\text { Se explicó a los estudiantes la investigación que se iba a realizar, las } \\
\text { características, la teoría de aprendizaje que se iba a utilizar y los objetivos } \\
\text { de la misma. }\end{array}$ \\
\hline 2 & Caracterización & $\begin{array}{l}\text { Se evaluó, a través de comunicación directa y evaluaciones impromptu, } \\
\text { la posible caracterización de los estudiantes en tres niveles: estudiantes } \\
\text { talentosos, estudiantes medianamente talentosos y estudiantes de bajo talento. }\end{array}$ \\
\hline 3 & Segmentación & $\begin{array}{l}\text { Se dividió el grupo de estudiantes en dos subgrupos ambos de } 11 \text { estudiantes. } \\
\text { En ambos grupos se procuró que quedaran igual número de estudiantes tanto } \\
\text { en cantidad como en caracterización de acuerdo a los resultados de la fase } 2 \text {. }\end{array}$ \\
\hline 4 & Paralelización & $\begin{array}{l}\text { Se establecieron horarios paralelos en ambos grupos de forma que se pudieran } \\
\text { explicar en dos sesiones el tema sistema binario. Como se cuenta con sesiones } \\
\text { de dos horas se realizó así: a un grupo se le expuso el tema con el método } \\
\text { tradicional de explicación magistral y en esa sesión se entregó todo el tema. } \\
\text { Al otro grupo (en la segunda hora), se le explicó bajo la metodología de } \\
\text { aprendizaje por descubrimiento. }\end{array}$ \\
\hline 5 & Evaluación & $\begin{array}{l}\text { Finalizadas las dos sesiones, se realizó la misma evaluación acerca del tema } \\
\text { en ambos grupos. }\end{array}$ \\
\hline 6 & Retroalimentación & $\begin{array}{l}\text { Se consultó la opinión de cada uno de los estudiantes en relación con la } \\
\text { metodología utilizada. }\end{array}$ \\
\hline 7 & Socialización & $\begin{array}{l}\text { Se entregaron los resultados de las evaluaciones y se socializó la experiencia } \\
\text { con los estudiantes. }\end{array}$ \\
\hline
\end{tabular}

Fuente: elaboración propia

Durante el curso, y específicamente en relación con la temática abordada, se adoptaron instrumentos de exposición, explicación y evaluación que permitieron evaluar, retroalimentar y detectar dudas en los estudiantes durante las sesiones en que el tema fue abordado. Vale la pena anotar que el detalle de los instrumentos se ha omitido en este artículo solamente por razones de extensión del mismo, para estar en sintonía con las políticas editoriales de la revista.

La interacción con cada uno de los grupos se realizó en horarios diferentes dividiendo la sesión de dos horas en dos sesiones de una hora cada una, de manera que cada subgrupo estuviera con el profesor sin la intervención ni participación del otro grupo y en las mismas condiciones ambientales, de modo, tiempo y lugar para recibir la exposición. La tabla 3 explica de una forma más detallada la metodología de exposición adoptada. 
Tabla 3. Metodología de exposición adoptada

\begin{tabular}{|c|c|c|c|}
\hline Grupo & Característica & Descripción & Evaluación \\
\hline 1 & $\begin{array}{l}\text { Grupo en el cual se aplicó la } \\
\text { metodología tradicional ex- } \\
\text { plicando el sistema binario } \\
\text { tal como se conoce. }\end{array}$ & $\begin{array}{l}\text { Se explicó el sistema binario tal } \\
\text { como se conoce: tema completo, } \\
\text { exposición magistral tradicio- } \\
\text { nal, conversión de binario a } \\
\text { decimal y viceversa. También } \\
\text { se hicieron ejercicios de suma } \\
\text { binaria. }\end{array}$ & $\begin{array}{l}\text { Se realizó una evaluación de } \\
\text { diez ejercicios con pregun- } \\
\text { tas sobre el sistema binario, } \\
\text { ejercicios de conversión de } \\
\text { binario a decimal y viceversa. } \\
\text { Además se plantearon ejerci- } \\
\text { cios de sumas binarias. }\end{array}$ \\
\hline 2 & $\begin{array}{l}\text { Grupo en el cual se aplicó } \\
\text { la teoría de aprendizaje por } \\
\text { descubrimiento en relación } \\
\text { con un sistema numérico } \\
\text { hipotético. }\end{array}$ & $\begin{array}{l}\text { Se explicó un sistema hipotético } \\
\text { llamado sistema quinquenario } \\
\text { cuya base es } 5 \text {, sus dígitos son } \\
0,1,2,3 \text { y } 4 \text {, su operación es la } \\
\text { suma y se hicieron ejercicios } \\
\text { de conversión con el sistema } \\
\text { decimal. Cuando el sistema } \\
\text { quinquenario fue asimilado, } \\
\text { se les planteó a los estudiantes } \\
\text { la idea de describir un sistema } \\
\text { cuya base fuera } 2 \text {, sus dígitos } 0 \text { y } \\
1 \text { y su operación fuera la suma. } \\
\text { Se les indicó que descubrieran } \\
\text { cómo sería la conversión de este } \\
\text { sistema al decimal y viceversa. }\end{array}$ & $\begin{array}{l}\text { Se realizó una evaluación con } \\
\text { diez ejercicios con pregun- } \\
\text { tas sobre el sistema binario, } \\
\text { ejercicios de conversión de } \\
\text { binario a decimal y viceversa. } \\
\text { Además se plantearon ejerci- } \\
\text { cios de sumas binarias. }\end{array}$ \\
\hline
\end{tabular}

Fuente: elaboración propia

\section{RESULTADOS}

Se realizaron pruebas escritas y una serie de evaluaciones tendientes a verificar la comprensión, interiorización y asimilación del concepto de lo que es un sistema numérico y, específicamente, el sistema binario. Se tomó como base de análisis los resultados cuantitativos de dichas evaluaciones tal como se explica a continuación.

Los resultados de la evaluación se presentan en la tabla 4 en donde se muestran las diferencias cuantitativas de los dos subgrupos dentro de cada uno de los grupos con los cuales se hizo la investigación. Debe anotarse que la evaluación en cada subgrupo se hizo manteniendo las mismas características de nivel y complejidad de preguntas y ejercicios. Se acudió a evaluar con cinco preguntas sobre el sistema numérico estudiado, tres ejercicios de conversión del sistema binario al sistema decimal, dos ejercicios de conversión del sistema decimal al sistema binario. 
Vale la pena tener en cuenta que, para los estudiantes con los cuales se adoptó la metodología tradicional, la evaluación sobre el sistema binario se convirtió en una forma de repaso de lo visto. En el caso del subgrupo de estudiantes en donde se adoptó como ejemplo el sistema quinquenario, el sistema binario se convirtió en un reto para descubrir a partir de las bases matemáticas vistas. Debe acotarse que se presentan en la tabla 4 los resultados de la evaluación realizada como prueba en relación con el tema del sistema binario.

Tabla 4. Evaluación del sistema binario

\begin{tabular}{|c|c|c|c|}
\hline$A \tilde{n} o$ & Semestre & Subgrupo & $\begin{array}{l}\text { Promedio del } \\
\text { grupo }\end{array}$ \\
\hline \multirow{4}{*}{2014} & \multirow{2}{*}{ I } & Método tradicional & 3,8 \\
\hline & & Método aprendizaje por descubrimiento & 4,4 \\
\hline & \multirow{2}{*}{ II } & Método tradicional & 3,5 \\
\hline & & Método aprendizaje por descubrimiento & 3,9 \\
\hline \multirow{4}{*}{2015} & \multirow{2}{*}{ I } & Método tradicional & 4,0 \\
\hline & & Método aprendizaje por descubrimiento & 4,2 \\
\hline & \multirow{2}{*}{ II } & Método tradicional & 4,1 \\
\hline & & Método aprendizaje por descubrimiento & 4,1 \\
\hline \multirow{4}{*}{2016} & \multirow{2}{*}{ I } & Método tradicional & 4,0 \\
\hline & & Método aprendizaje por descubrimiento & 4,3 \\
\hline & \multirow{2}{*}{ II } & Método tradicional & 3,8 \\
\hline & & Método aprendizaje por descubrimiento & 4,1 \\
\hline
\end{tabular}

Fuente: elaboración propia

Vale la pena tener en cuenta que, en todos los grupos, el promedio del subgrupo con quienes se compartió el conocimiento del sistema binario a partir de la metodología tradicional siempre obtuvo un promedio menor al subgrupo con el cual se adoptó la metodología de aprendizaje por descubrimiento. En el caso del II semestre del año 2015, el promedio del grupo con metodología tradicional fue de 4,13 y el promedio del grupo con metodología de aprendizaje por descubrimiento fue de 4,19. Para la presentación de datos se adoptó solamente un dígito decimal.

La retroalimentación del proceso se realizó por escrito a partir de tres preguntas abiertas: 1) ¿Cómo le pareció la metodología utilizada?, 2) ¿Qué ventajas encontró en la metodología?, 3) ¿Hubiera querido estar en el otro grupo? La tabla 5 presenta algunas de las respuestas. 
Tabla 5. Descripción de algunas respuestas

\begin{tabular}{|c|c|c|c|c|}
\hline Grupo & Descripción & Pregunta 1 & Pregunta 2 & Pregunta 3 \\
\hline 1 & $\begin{array}{l}\text { Método } \\
\text { tradicional }\end{array}$ & $\begin{array}{l}\text { - Me pareció buena la } \\
\text { explicación del profe, } \\
\text { pero lo vi todo normal } \\
\text { - El tema es sencillo, } \\
\text { pero es más fácil cuan- } \\
\text { do se lo explican a uno } \\
\text { así } \\
\text { - La clase fue muy nor- } \\
\text { mal }\end{array}$ & $\begin{array}{l}\text { - El profesor explica } \\
\text { muy bien } \\
\text { - Es la metodología que } \\
\text { conocemos } \\
\text { - El profe es muy orga- } \\
\text { nizado en el tablero } \\
\text { - Con una buena expli- } \\
\text { cación es suficiente }\end{array}$ & $\begin{array}{l}\text { - Me habría gustado es- } \\
\text { tar en el otro grupo } \\
\text { - Si hubiera querido } \\
\text { - Me han comentado } \\
\text { maravillas del otro sis- } \\
\text { tema } \\
\text { - Absolutamente sí }\end{array}$ \\
\hline 2 & $\begin{array}{l}\text { Método } \\
\text { Aprendizaje } \\
\text { por descu- } \\
\text { brimiento }\end{array}$ & $\begin{array}{l}\text { - Me pareció muy in- } \\
\text { teresante que uno se } \\
\text { imagine un sistema hi- } \\
\text { potético } \\
\text { - Se me hizo muy fácil } \\
\text { pasar del sistema quin- } \\
\text { quenario al sistema bi- } \\
\text { nario } \\
\text { - Al fin juff! (sic) pude } \\
\text { entender el sistema bi- } \\
\text { nario }\end{array}$ & $\begin{array}{l}\text { - Le permite a uno avan- } \\
\text { zar por cuenta propia } \\
\text { - El profe es un verraco } \\
\text { muy bueno (sic) } \\
\text { - Descubrir es lo mejor } \\
\text { que me ha pasado } \\
\text { - Entendí todo } \\
\text { - Inmejorable la meto- } \\
\text { dología }\end{array}$ & $\begin{array}{l}\text { - Para nada, descubrir } \\
\text { es muy interesante } \\
\text { - No me hubiera gusta- } \\
\text { do } \\
\text { - Ojalá esta metodología } \\
\text { la usaran en todas las } \\
\text { materias } \\
\text { - No hubiera querido es- } \\
\text { tar en el otro grupo }\end{array}$ \\
\hline
\end{tabular}

Fuente: elaboración propia

\section{DISCUSIÓN}

En referencia con la estrategia adoptada, puede decirse que la explicación pretende alterar la teoría original para que, a partir de ella, el estudiante se oriente en la búsqueda de la teoría real, es decir, la que corresponde al sistema binario como tal. El aprendizaje de un sistema hipotético le plantea al estudiante la posibilidad de comprender lo que es un sistema numérico de base 5 pero, al tiempo, le provee algunas herramientas matemáticas y conceptuales para que se arriesgue a inferir las características de un sistema numérico de base $\mathrm{n}$ que, para el caso del sistema binario, corresponde a un sistema base 2 .

Una de las bondades que se pueden capitalizar de la teoría del aprendizaje por descubrimiento, radica en el hecho de poner a disposición de los estudiantes, conceptos que van más allá del conocimiento real que se quiere alcanzar, esto es (como sucede en este caso), ir de un sistema hipotético base 5 a un sistema verdadero base 2, heredando conceptualmente fundamentos teóricos y extrapolándolos a través de un proceso de 
simplificación de forma que se puedan asimilar por el camino del descubrimiento, base para que el conocimiento pueda perdurar en un plazo más extenso con significado.

En cuanto a la evaluación realizada sobre el sistema binario, se nota que el promedio en el subgrupo al cual se le aplicó la metodología de aprendizaje por descubrimiento es cuantitativamente más alto que el promedio del subgrupo en el cual se aplicó una metodología tradicional. Esto hace suponer que la mejora en el resultado cuantitativo, si se entiende que era la misma evaluación (en cuanto a cantidad de preguntas, condiciones, calidad y complejidad de las mismas), podría ser producto de la metodología aplicada pues pareciera confirmarse que los estudiantes le confieren sentido y significado a lo que ellos mismos descubren.

Las reflexiones a este respecto se develan en las respuestas cualitativas personales de cada estudiante que permiten inferir lo que los resultados cuantitativos parecieran decir. Si bien en términos generales las opiniones son, de alguna manera, favorables, en la metodología tradicional se puede advertir que las respuestas destacan mucho más las cualidades propias del docente mientras que en la metodología con aprendizaje por descubrimiento las respuestas destacan, y hacen hincapié, en la metodología como tal. En el caso de la tabla 5 se muestran tan solo algunas de las respuestas, escogidas completamente al azar, pero el conjunto completo de las respuestas confirma, aún más, lo que esta selección pareciera indicar.

Se ha planeado realizar pruebas similares en cursos equivalentes y posteriores a la presentación de este artículo, es decir, en los cursos equivalentes de los semestres I y II del año 2016 con el ánimo de permitir que los datos digan lo suyo y, de esta forma, tener más elementos de juicio para realizar otras inferencias. Por las condiciones metodológicas de la presente investigación, los resultados pueden considerarse confiables, aunque hacer esta experiencia en los semestres posteriores permitiría solidificar dichas conclusiones y profundizar en otras.

Los resultados cuantitativos de la tabla 4 evidencian un camino muy interesante en relación con el proceso de aprendizaje, pues se tuvo especial cuidado en que las evaluaciones fueran equivalentes en nivel de exigencia y en complejidad y, sin embargo, los resultados obtenidos favorecen significativamente a los estudiantes con quienes se adoptó la metodología basada en aprendizaje por descubrimiento. Sabiendo que el promedio es una medida de tendencia central que se afecta por los valores extremos, a pesar de ellos y manteniéndolos, se observan unos resultados cuantitativos más favorables que en los subgrupos donde se adoptó el método tradicional.

Por su parte, en lo que corresponde a las opiniones, los resultados parecieran indicar que para el estudiante resulta ser un reto muy interesante poder utilizar el nuevo 
conocimiento adquirido para aplicarlo en evaluaciones cuyo corpus sea alcanzable por él mismo. Tal como lo indica la teoría del aprendizaje por descubrimiento, el aprendizaje se logra siempre que exista un marco coherente de premios y castigos y, en el caso de la evaluación adoptada, el éxito está en que las preguntas, ejercicios y conversiones entre sistemas numéricos estén al alcance de los alumnos a partir del conocimiento impartido sobre el hipotético sistema quinquenario, de forma que puedan concebir otro sistema numérico con el simple hecho de cambiar la base del mismo.

\section{CONCLUSIONES}

Es posible encontrar metodologías que propicien el acceso al conocimiento y una ubicación del mismo en la memoria a largo plazo a partir de la teoría de aprendizaje por descubrimiento, que hagan factible, además, la apropiación, asimilación, aplicación y retroalimentación de conceptos matemáticos, técnicos o científicos en el desarrollo de un proceso de formación profesional. Los resultados obtenidos sugieren la necesidad de conocer teorías, aplicaciones y estrategias de aprendizaje en programas de ingeniería y específicamente en el área de programación de computadores.

En procesos de investigación científica y tecnológica dentro del marco educativo, es muy importante, cuando se quieren realizar experiencias paralelas, que se mantengan las mismas condiciones de todo tipo en los grupos que se quieran analizar [21] dado que cualquier cambio, por ejemplo, en el clima o en la hora de la clase puede ser determinante para que los resultados de investigaciones de este tipo estén sesgados por factores aparentemente insignificantes pero investigativamente determinantes. En este sentido, tal como los resultados lo indican, se procuró que se cumpliera plenamente en el desarrollo de la presente investigación.

Siempre pareciera necesario realizar evaluaciones objetivas que confirmen lo aprendido por los estudiantes. Se hace necesario que los resultados de este tipo de investigaciones se socialicen con los estudiantes que participan en ellas para que sean ellos mismos los que retroalimenten y enriquezcan con sus comentarios, criticas, observaciones y posibles correcciones el proceso como tal.

Finalmente, no se pueden desconocer las bondades de la teoría de aprendizaje por descubrimiento como base para aproximar a los estudiantes de ingeniería al conocimiento tecnológico y científico, bien sea que efectivamente descubran nuevo conocimiento o bien que crean que lo han descubierto. Lo más importante de este proceso investigativo es que los estudiantes aprendieron a programar y esto se puede concluir por concepto cualitativo y observación directa del docente en un porcentaje significativamente alto (más del $80 \%$ ). Los detalles se han omitido por extensión del artículo. 
El desarrollo de proyectos de investigación educativo implica un fundamento teórico y científico que posibilite confiar en las inferencias desde una perspectiva confiable y por ello conviene documentarse apropiadamente [22], bien sea en el desarrollo de proyectos investigativos dentro del área puramente educativa y formativa o dentro de procesos de aprendizaje de otras áreas en donde, posiblemente, sea donde más se necesiten [23].

La metodología aplicada y el sustento a partir de la teoría de aprendizaje por descubrimiento, permiten concluir que el proceso ha sido exitoso y que los estudiantes han aprendido a interiorizar y a asimilar el concepto de un sistema numérico y, específicamente, las características del sistema binario, base fundamental para la comprensión de las tecnologías computacionales modernas dentro del contexto del proceso de formación de ingenieros de sistemas.

\section{REFERENCIAS}

[1] B, Joyce, M. Weil, y E. Calhoun, (2014). Models of Teaching 9 ed., Nueva York: Pearson.

[2] I. Milne, y G. Rowe, "Difficulties in learning and teaching programming- Views of students and tutors," Educationa and Information technologies, vol. 7, N. ${ }^{\circ}$ 1, 55-66, 2002

[3] A. Vega Escobar y A. Espinel Ortega, "Aspectos fundamentales para la enseñanza de la programación básica en ingeniería," Revista Avances en Sistemas e Informática, vol. 7, N. 1 , $7-13,2010$

[4] D. Jackson, D. y R.Miller, [En línea], acceso 2 de febrero, 2009: Disponible: http://people. csail.mit.edu/dnj/articles/teaching-6005.pdf

[5] J. Kaasboll, Exploring didacti models for programming. Oslo: Universidad de Oslo. 1999.

[6] H. Paz Penagos, "Aprendizaje autónomo y estilo cognitivo: diseño didáctico, metodología y evaluación,” Revista Educación en Ingeniería, vol. 9, N.` 17, pp. 53-65, 2014

[7] F. Sally, "What are we doing when we teach programming?" presentado en FIE'99: Frontiers in Education. 29th Annual Frontiers in Education Conference. Designing the Future of Science and Engineering Education, Sesión 12a sesión. San Juan de Puerto Rico, Puerto Rico, 1999.

[8] T. Koulouri, S. Lauria y R. Macredie, "Teaching introductory programming: a quantitative evaluation of differets approaches," ACM Transactions on Computing Education, 14, N. ${ }^{4}$, 2015

[9] C. Romero Chaves y M. Rosero Sosa, "Modelo de enseñanza y su relación con los procesos metacognitivos en programación de sistemas," Revista Educación en Ingeniería, vol. 3, 2014

[10] O. Trejos Buriticá, Significado y competencias, Pereira: Papiro, 2013.

[11] J. Bruner, Actos de significado: mas allá de la revolución cognitiva, Boston:Alianza Editorial, 2009. 
[12] M. Guerrero Sánchez, Metodologías activas y aprendizaje por descubrimiento: las TIC y la educación, Boston: Amazon Digital Services, 2014.

[13] D. Ausubel,Psychology of meaningful verbal learning: an introduction to school learning, Nueva York: Grune \& Straton, 1963.

[14] Unesco, "Estándares de competencia en TIC para docentes," [En línea], acceso junio, 2016: Disponible: http://www.eduteka.org/EstandaresDocentesUnesco.php

[15] E, Piza, Los números reales y sus principales subconjuntos: construcciones y axiomatización de los sistemas numéricos. Madrid: Editorial Académica Española, 2012.

[16] J. Coronado Padilla, Sistemas numéricos residuales: fundamentos lógicomatemáticos. Bogotá: Universidad de la Salle, 2012.

[17] R. Ziveri, R. y P. Galassi, Sistema binario (Edición italiana)), Roma: Abel Books, 2013.

[18] J. Jiménez Murillo, Matemáticas para la computación. Ciudad de México: Alfaomega, 2014.

[19] J. S. Bruner, El proceso de la educación, Ciudad de México: Editorial Hispanoamericana, 1963.

[20] S. Campillay y S. Meléndez, "Análisis de impacto de metodología activa y aprendizaje en asignaturas de Ingeniería," Actividades Investigativas en Educación, vol. 15, N. 2, 2015

[21] R. Bisquerra Alcina, Metodología de la investigación educativa. Madrid: Editorial La Muralla S. A., 2004.

[22] A. Díaz-Barriga, et al., Metodología de la investigación educativa. Ciudad de México: Editorial Diaz de Santos, 2014.

[23] D. Begoña García, et al., Fundamentos Básicos de Metodología de la Investigación Educativa. Bogotá: Editorial CCS, 2012. 